\title{
Otimização da prensagem de grãos de girassol e sua caracterização
}

\author{
Anna L. M. T. Pighinelli' ${ }^{1}$ Kil J. Park ${ }^{1}$, Ana M. Rauen ${ }^{2}$ \& Rafael A. de Oliveira ${ }^{1}$
}

\begin{abstract}
RESUMO
A prensagem mecânica contínua de oleaginosas se destaca por sua rapidez, facilidade de manuseio e baixo custo de instalação e manutenção adequando-se perfeitamente à realidade das pequenas comunidades rurais; quanto à eficiência, a extração por solvente ainda é melhor. Para melhorar o desempenho, pesquisadores observaram a influência de variáveis no rendimento em óleo, como o teor de umidade e temperatura dos grãos e aspectos construtivos da prensa. Quanto ao preparo inicial da matéria-prima, estudos mostram que existe uma faixa ótima de teor de umidade, além de valores de temperatura que possam facilitar ou prejudicar a fluidez do óleo. Avaliou-se, no presente trabalho, a influência da temperatura e do teor de umidade dos grãos de girassol no rendimento em óleo bruto obtido pela extração mecânica. Para a faixa de trabalho escolhida a umidade e a interação umidade $x$ temperatura foram significativos afetando, de forma negativa, o rendimento para um nível de confiança de $95 \%$. O maior rendimento em óleo bruto foi para umidade na faixa de 8 a 8,5\% e temperatura entre 47,8 a $60^{\circ} \mathrm{C}$. O óleo bruto apresentou características semelhantes as do óleo de girassol comercial obtido via extração por solvente.
\end{abstract}

Palavras-chave: superfície de resposta, teor de umidade, temperatura, rendimento em óleo

\section{Optimization of sunflower grain pressing and its characterization}

\begin{abstract}
The continuous screw pressing of oilseeds is noted for its speed, case of handling and for its low cost installation and support, adapting perfectly to small agricultural communities. However, in terms of efficiency, the traditional solvent extraction still has a higher yield. Attempting to improve the screw pressing technique, some researchers had observed that some variables had an influence on oil yield, such as grain moisture content, grain temperature and some construction aspects of the press. In relation to the initial raw material preparation, studies reveal the possibility of finding optimal values of the moisture content and temperature. In this work, the influence of temperature and moisture content on oil yield was evaluated. For the experimental range of values chosen, among the effects studied, the moisture content and the interaction between moisture and temperature were significant, affecting in a negative way the oil yield, to $95 \%$ of confidence. The biggest value of oil yield was reached with the moisture content between 8 to $8.5 \%$ and the temperatures between 47.8 to $60^{\circ} \mathrm{C}$. The oil obtained by pressing presented characteristics similar to those of commercial sunflower oil.
\end{abstract}

Key words: response surface, moisture content, temperature, oil yield

FEAGRI/ UNICAMP: Cidade Universitária Zeferino Vaz, s/n, CP 6011. CEP 13083-875 Campinas, SP. Fone: (19) 3521-1076, fax: (19) 3521-1010. E-mail: annalets@agr.unicamp.br; kil@agr.unicamp.br; augustus@agr.unicamp.br

2 ITAL. Av. Brasil, 2880, CP 139. CEP 13070-178 Campinas, SP. Fone: (19) 3743-1782. E-mail: anarauen@ital.sp.gov.br 


\section{INTRODUÇÃO}

Os óleos vegetais se têm destacado pela possibilidade de uso na obtenção de biodiesel, combustível renovável e substituto parcial do óleo diesel. Visando atender as pequenas comunidades rurais, é necessário investir em tecnologias alternativas de extração de óleos vegetais, como as prensas mecânicas contínuas. As pesquisas sobre este tema estão sendo retomadas buscando-se a melhoria do processo contínuo; a prensa mecânica contínua ou expeller é um método simples, adaptável a diversos tipos de oleaginosas e, em curto espaço de tempo, permite a instalação em pequenas propriedades e o uso do subproduto (torta) como adubo ou ração animal (Singh \& Bargale, 2000).

A eficiência na extração está por demais relacionada ao aquecimento e teor de umidade dos grãos. O aquecimento quebra as células de óleo facilitando sua saída e o teor de umidade é o fator que mais afeta a quantidade de óleo residual na torta (Singh et al., 1984; Singh et al., 2002).

As prensas de pequena capacidade $\left(40 \mathrm{~kg} \mathrm{~h}^{-1}\right)$ são, em geral, ineficientes, deixando em torno de 8 a 14\% de óleo na torta (Srikahtha, 1980). A otimização das variáveis envolvidas no processo, temperatura e teor de umidade das amostras, auxilia no aumento do rendimento (Ohlson, 1992).

O teor de umidade ótimo deve ser encontrado: valores muito altos reduzem a fricção, causam baixo rendimento e valores muito baixos prejudicam o funcionamento da prensa (Reuber, 1992; Singh \& Bargale, 1990). Singh et al. (2002) mostraram que uma diminuição no teor de umidade e aumento na temperatura melhoram o rendimento em óleo.

A metodologia da superfície de resposta (RSM) é uma coleção de técnicas estatísticas e matemáticas usadas para desenvolver, melhorar e otimizar processos, dimensionar e formular produtos, além de amplamente utilizadas na indústria, onde são muitas as variáveis de entrada (variáveis independentes) que, por sua vez, influenciam uma resposta (variável dependente), como o rendimento do produto ou do processo (Myers \& Montgomery, 2002).

Séries de estratégias experimentais são utilizadas para explorarem o espaço das variáveis independentes, que são modeladas de forma a se obter uma relação aproximada entre elas e a resposta desejada. Uma estratégia benquista é o planejamento experimental, em vários níveis, permitindo boa correlação entre as variáveis independentes e as respostas (Myers \& Montgomery, 2002).

De acordo com Barros Neto et al. (2003), a RSM se compõe de duas etapas: modelagem e deslocamento, em que a primeira ajusta modelos lineares ou quadráticos a resultados experimentais obtidos de planejamentos experimentais e a segunda busca o caminho de máxima inclinação de um modelo, que é o caminho no qual a resposta varia de forma mais pronunciada.

Se $n$ variáveis controladas estão envolvidas, o planejamento necessita de $2^{\text {n }}$ ensaios diferentes. Calculam-se os efeitos principais e de interação das variáveis independentes sobre as respostas, determinando aqueles mais significativos e os comparando com o valor do efeito e erro experimental esti- mado. Quando o modelo de primeira ordem não for eficiente, realizar-se-ão ensaios nos pontos axiais para um modelo de segunda ordem (planejamento experimental do tipo central composto).

A obtenção dos modelos empíricos por regressões lineares e não-lineares segue uma análise de variância utilizando-se, como parâmetros, coeficiente de determinação $\left(\mathrm{R}^{2}\right)$ e valor estimado do teste F (Box et al., 1978); a análise dos resíduos também é importante para avaliar a qualidade do ajuste, enquanto valores residuais altos indicam má qualidade no ajuste do modelo (Barros Neto et al., 2003).

O girassol (Helianthus annus L.) é muito utilizado nas rotações de culturas como reciclador de nutrientes atacando aleopaticamente, as plantas invasoras, melhorando as características físicas do solo; esta versatilidade torna a cultura adequada para pequenos produtores; além das vantagens relacionadas à rotação de culturas, o girassol é ótimo para a produção de mel, grãos e óleo (Ungaro, 2001); o subproduto da extração a frio (torta) é usado em rações animais e adubo, contendo $40 \%$ de proteína, teor de óleo entre 7 e 9\% na extração mecânica (Weiss, 1983).

Com o presente trabalho visou-se otimizar o processo de prensagem mecânica contínua de uma mini-prensa, em função de duas variáveis independentes (temperatura e teor de umidade) tendo como resposta o rendimento em óleo bruto e sua caracterização.

\section{MATERIAL E MÉTODOS}

Utilizaram-se, no planejamento experimental, duas variáveis independentes (teor de umidade e temperatura dos grãos) e como variável dependente (resposta), o rendimento em óleo bruto, com o qual se avaliou experimentalmente o comportamento das variáveis independentes na resposta, seguindo-se o planejamento fatorial completo (com 7 experimentos), mostrado na Tabela 1 .

Tabela 1. Planejamento fatorial completo da prensagem dos grãos de girassol

\begin{tabular}{|c|c|c|c|c|c|}
\hline \multirow{2}{*}{ Ensaios } & & \multicolumn{2}{|c|}{ Variável codificada } & \multicolumn{2}{|c|}{ Variável real } \\
\hline & & $X_{1}$ & $x_{2}$ & $\mathrm{~T}\left({ }^{\circ} \mathrm{C}\right)$ & U (\%) \\
\hline \multirow{4}{*}{ Pontos Fatoriais } & 1 & -1 & -1 & 25 & 8,0 \\
\hline & 2 & +1 & -1 & 60 & 8,0 \\
\hline & 3 & -1 & +1 & 25 & 12,0 \\
\hline & 4 & +1 & +1 & 60 & 12,0 \\
\hline \multirow{3}{*}{ Pontos Centrais } & 5 & 0 & 0 & 42,5 & 10,0 \\
\hline & 6 & 0 & 0 & 42,5 & 10,0 \\
\hline & 7 & 0 & 0 & 42,5 & 10,0 \\
\hline
\end{tabular}

A resposta (rendimento em óleo bruto) foi obtida por um modelo contendo termos de primeira ordem e de interação entre os termos, conforme a Eq. 1.

$$
\mathrm{REND}=\mathrm{b}_{0}+\mathrm{b}_{1} * \mathrm{X}_{1}+\mathrm{b}_{2} * \mathrm{X}_{2}+\mathrm{b}_{12} * \mathrm{X}_{1} * \mathrm{X}_{2}
$$

A variável de resposta medida é REND, que foi analisada 
por múltipla regressão através do método em que se utiliza erro padrão para ajustar a Eq. 1, no software estatístico Statistica versão 6.0 (2001).

\section{Preparo das amostras de grãos}

Os grãos de girassol com 6,4\% de umidade (base seca, bs), foram adquiridos no comércio local e são da variedade IAC Iarama, caracterizada por grãos escuros com cerca de $40 \%$ de óleo. Para adequação da matéria-prima aos vários teores de umidade do planejamento experimental as amostras foram separadas, umidificadas com a quantidade necessária de água e acondicionadas em plásticos lacrados em câmara fria a $10^{\circ} \mathrm{C}$ até atingirem o equilíbrio (aproximadamente 10 dias).

\section{Extração do óleo}

Fez-se por prensagem a frio em prensa do tipo expeller, modelo MPE-40, marca ECIRTEC, a extração do óleo com processamento de 40 a $60 \mathrm{~kg} \mathrm{~h}^{-1}$ de matéria-prima.

Os grãos foram retirados da câmara fria, deixados a temperatura ambiente e posteriormente aquecidos até as temperaturas estabelecidas no planejamento experimental; este aquecimento foi feito em aquecedor por resistência elétrica e com sistema de mistura da massa de grãos.

Após atingirem a temperatura do planejamento, os grãos foram colocados na prensa; terminado o processamento, o óleo bruto, antes da filtração, e as tortas obtidas, foram pesados; o óleo seguiu para filtração em papel de filtro e posteriores análises laboratoriais, enquanto a torta foi analisada em relação ao teor de lipídios.

\section{Análises de caracterização}

Definiu-se o teor de umidade dos grãos pelo método da estufa (marca FANEM modelo 320-SE) durante 24 h, a temperatura de $105^{\circ} \mathrm{C}$, de acordo com a AOAC (1998). Todas as análises a seguir foram feitas seguindo as normas da AOCS (1998).

O teor de lipídios foi obtido nos grãos e na torta resultante da prensagem, extraindo-se sua matéria graxa por éter de petróleo em um extrator tipo BUTT (extração direta); o método utilizado para a operação foi o Bc 3-49.

Fez-se a determinação da cor das amostras de óleo por comparação com padrões de cores seguindo-se a metodologia oficial Cc 13b-45, e as leituras feitas no tintômetro Lovibond.

O índice de refração de um óleo está relacionado com o grau de saturação das ligações, com o teor de ácidos graxos livres, oxidação e tratamento térmico da amostra, seguindose o método oficial Cc 7-25; as leituras foram feitas em refratômetro resfriado com banho para manter a temperatura de $40{ }^{\circ} \mathrm{C}$. Os ácidos graxos livres existentes na amostra foram determinados por titulometria, como indicado no método Ca $5 a-40$.

O índice de iodo foi determinado pelo Método de Wijs conforme o método Cd 1-25, ele mede a insaturação de óleo e gorduras e é expresso em termos do número de centigramas de iodo absorvidos por grama de amostra (percentagem de iodo absorvido). O índice de saponificação é a quantidade de álcali necessária para saponificar uma quantidade definida de amostra. É expresso como o número de miligramas de hidró- xido de potássio $(\mathrm{KOH})$ suficiente para saponificar $1 \mathrm{~g}$ de amostra; enfim, a determinação é realizada conforme método oficial Cd 3-25; por outro lado, o índice de peróxido determina todas as substâncias que oxidam o iodeto de potássio (KI), em miliequivalentes de peróxido por 100 g de amostra, sob as condições de teste; essas substâncias são, geralmente, consideradas peróxidos ou outros produtos similares da oxidação de gorduras. O método para esta análise é o Cd 8-53.

A densidade relativa de um óleo determina a relação de peso de um volume unitário da amostra a $20{ }^{\circ} \mathrm{C}$ para o peso de um volume unitário da água a $20^{\circ} \mathrm{C}$, conforme mostrado no método oficial Cc 10a-25.

Nas matérias insaponificáveis se incluem aquelas substâncias freqüentemente encontradas dissolvidas nos óleos e gorduras, as quais não se saponificam com os álcalis cáusticos mas são solúveis em solventes comuns de gorduras e estão incluídos álcoois alifáticos, esteróis, pigmentos e hidrocarbonetos. O método oficial é o Ca 6a-40.

Fez-se a composição em ácidos graxos em cromatógrafo gasoso, conforme métodos Ce 1e-91; Ce 1F-96; Ce 1-62 e Ce 2-66 da AOCS (1998) e método 996.06 da AOAC (1998).

\section{RESULTADOS E DISCUSSÃO}

Na Tabela 2 estão os rendimentos em óleo bruto obtidos com a extração do óleo de girassol. Os valores entre parênteses correspondem aos pontos do planejamento experimental.

Tabela 2. Rendimento da extração de óleo bruto de girassol

\begin{tabular}{|c|c|c|c|c|}
\hline \multirow{2}{*}{ Ensaios } & & \multicolumn{2}{|c|}{ Variável real } & \multirow{2}{*}{$\begin{array}{l}\text { Rendimento em } \\
\text { óleo bruto (\%) }\end{array}$} \\
\hline & & $\mathrm{T}\left({ }^{\circ} \mathrm{C}\right)$ & $U(\%)$ & \\
\hline \multirow{4}{*}{ Pontos Fatoriais } & 1 & $25(-1)$ & $8,0(-1)$ & 59,90 \\
\hline & 2 & $60(1)$ & $8,0(-1)$ & 64,44 \\
\hline & 3 & $25(-1)$ & $12,0(1)$ & 55,21 \\
\hline & 4 & 60 (1) & $12,0(1)$ & 49,19 \\
\hline \multirow{3}{*}{ Pontos Centrais } & 5 & $42,5(0)$ & $10,0(0)$ & 55,56 \\
\hline & 6 & $42,5(0)$ & $10,0(0)$ & 55,76 \\
\hline & 7 & $42,5(0)$ & $10,0(0)$ & 58,85 \\
\hline
\end{tabular}

Analisando-se a triplicata do ponto central, obteve-se um coeficiente de variação de 3,25\% indicando que os experimentos apresentaram boa precisão possíveis, portanto, de serem repetidos.

Na Tabela 3 se encontram os efeitos lineares dos parâmetros e suas interações na avaliação do rendimento em óleo bruto; os valores em negrito indicam que o fator ou a interação é significativo para um nível de confiança de 95\% ( $\leq \leq 0,05)$.

Tabela 3. Efeito estimado, erro padrão e grau de significância estatística (p) para o rendimento da extração de óleo bruto de girassol

\begin{tabular}{lccc}
\hline Variáveis & Efeito & Erro padrão & $\mathbf{p}$ \\
Temperatura T (linear) & $-0,738771$ & 1,548143 & 0,6658 \\
Umidade U (linear) & $-9,967721$ & 1,548143 & 0,0076 \\
Interação T x U & $-5,285052$ & 1,548143 & 0,0420 \\
\hline
\end{tabular}


Pode-se observar, para o rendimento em óleo bruto, que o efeito linear da temperatura não influenciou significativamente na resposta; desta forma, o termo não-significativo foi retirado e os dados foram novamente analisados; a variável com maior efeito foi o termo linear da umidade, que atua de forma negativa indicando que, quanto menor a umidade dos grãos maior será o rendimento em óleo bruto. O outro termo significativo foi o da interação umidade $\mathrm{x}$ temperatura, que afeta o rendimento negativamente.

A Análise de Variância mostrada na Tabela 4 indica que o modelo de regressão gerado foi significativo (F-calculado para a regressão maior que o F-tabelado) e não houve falta de ajuste do modelo gerado (F-calculado para a falta de ajuste menor que o F-tabelado), conforme explicado por Barros Neto et al. (2002).

Tabela 4. Análise de Variância (ANOVA) para a extração de óleo de girassol

\begin{tabular}{lrccccc}
\hline $\begin{array}{l}\text { Fonte de } \\
\text { variação }\end{array}$ & $\begin{array}{c}\text { Soma } \\
\text { quadrática }\end{array}$ & $\begin{array}{c}\text { Graus de } \\
\text { liberdade }\end{array}$ & $\begin{array}{c}\text { Média } \\
\text { quadrática }\end{array}$ & Fcalc & Ftab & $\mathbf{R}^{2}$ \\
Regressão & $1,27 \mathrm{E}+02$ & 2 & $6,36 \mathrm{E}+01$ & 32,91 & 6,94 & 0,94271 \\
Resíduo & $7,74 \mathrm{E}+00$ & 4 & $1,93 \mathrm{E}+00$ & & & \\
Falta de ajuste & $9,15 \mathrm{E}-01$ & 3 & $3,05 \mathrm{E}-01$ & 0,04 & 215,71 & \\
Erro puro & $6,82 \mathrm{E}+00$ & 1 & $6,82 \mathrm{E}+00$ & & & \\
Total & $1,35 \mathrm{E}+02$ & 6 & & & & \\
\hline
\end{tabular}

É possível notar, na Figura 1 é possível observar a correlação entre os valores preditos pelo modelo e os valores observados; o gráfico indica uma aleatoriedade, confirmando que não houve formação de tendência na distribuição dos valores preditos pelo modelo; e com isto, pode-se afirmar que os resíduos não apresentaram comportamento sistemático fazendo com que o modelo seja adequado.

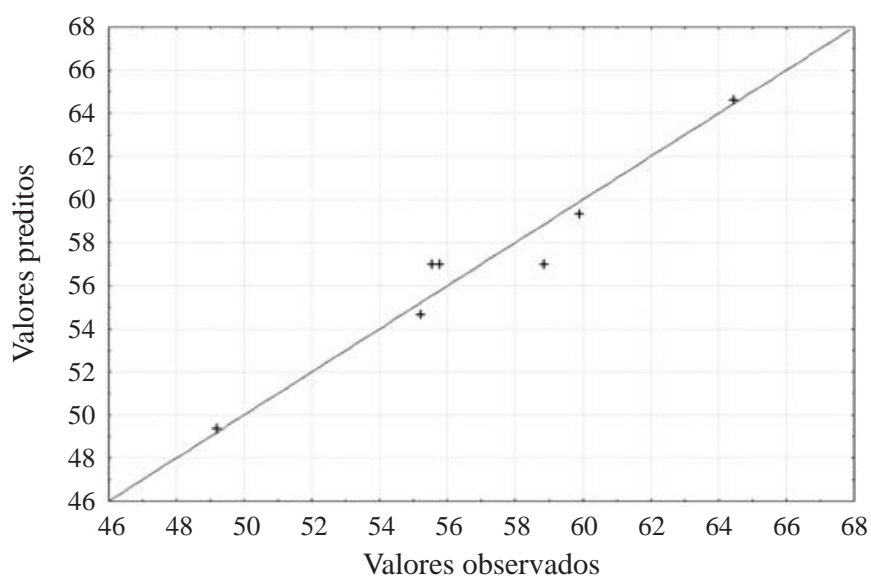

Figura 1. Valores preditos pelo modelo gerado comparado aos valores observados experimentalmente para a extração do óleo de girassol

Através das análises de regressão múltipla dos dados experimentais, obteve-se o modelo polinomial de primeira ordem, na forma não-codificada (Eq. 2). A análise dos resultados indicou que o modelo é mesmo linear e não existe a necessidade de se expandir o planejamento para um central composto, com adição dos pontos axiais.
REND $=56,9876-4,9839 * \mathrm{U}-2,6425 * \mathrm{U} * \mathrm{~T}$

Considerando-se que o desvio relativo médio entre os valores calculados pelo modelo e os valores experimentais foi de $4,77 \%$, que o coeficiente de determinação $\left(R^{2}\right)$ foi de $94,3 \%$ e que o teste $\mathrm{F}$ foi válido, pode-se dizer que, realmente, o modelo é preditivo para as condições do processo estudadas e, desta maneira, a superfície de resposta e as curvas em nível foram geradas (Figuras 2A e B).

A.

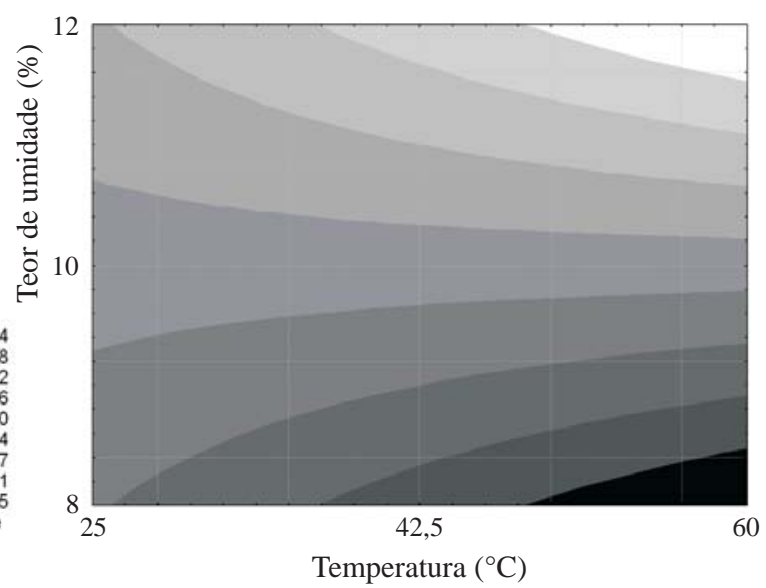

B.

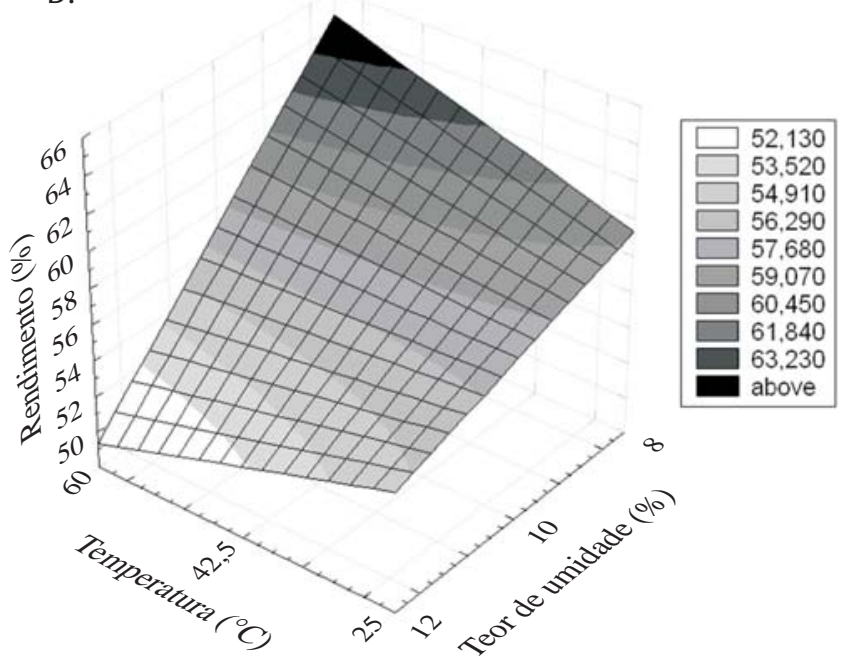

Figura 2. Curvas em nível e superfície de resposta para a prensagem mecânica contínua dos grãos de girassol

Na superfície de resposta fica fácil evidenciar o efeito da interação entre os fatores analisados e o efeito negativo da umidade no rendimento em óleo bruto.

A comparação entre a composição em ácidos graxos do óleo de girassol bruto e o óleo de girassol comercial (extraído industrialmente por solvente) está mostrada na Tabela 5 e, na Tabela 6 se acham as demais análises de caracterização, também se comparando os dois tipos de óleo.

Tanto a composição em ácidos graxos como as outras análises de caracterização, mostraram um óleo bruto muito 
Tabela 5. Composição em ácidos graxos do óleo de girassol

\begin{tabular}{|c|c|c|}
\hline Ácidos graxos & $\begin{array}{c}\text { Concentração óleo } \\
\text { bruto }(\%)\end{array}$ & $\begin{array}{c}\text { Concentração óleo } \\
\text { comercial }(\%)^{*}\end{array}$ \\
\hline C14:0 Mirístico & 0,1 & 0 \\
\hline C16:0 Palmítico & 7,0 & 6 \\
\hline C18:0 Esteárico & 3,1 & 4 \\
\hline 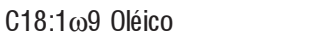 & 14,6 & 18 \\
\hline 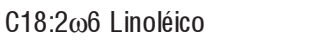 & 73,9 & 70 \\
\hline C20:0 Araquídico & 0,2 & - \\
\hline 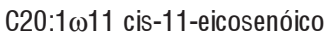 & 0,1 & - \\
\hline C18:3 $\omega 3 \alpha$ Alfa linolênico & 0,1 & - \\
\hline C22:0 Behênico & 0,7 & traços \\
\hline C24:0 Lignocérico & 0,2 & - \\
\hline
\end{tabular}

Tabela 6. Caracterização do óleo bruto de girassol

\begin{tabular}{lcc}
\hline Análise & Óleo bruto & Óleo comercial ${ }^{\star}$ \\
Índice de iodo - Método de Wijs $(\mathrm{cg} / \mathrm{g})$ & 148 & $125-136$ \\
Índice de refração $\left(40^{\circ} \mathrm{C}\right)$ & 1,4700 & $1,4663-1,6840$ \\
Índice de saponificação $(\mathrm{mg} \mathrm{KOH} / \mathrm{g})$ & 186,0 & $188,0-194,0$ \\
Matéria insaponificável $(\mathrm{g} / 100 \mathrm{~g})$ & 0,7 & $0,3-1,5$ \\
Densidade relativa $\left(20^{\circ} \mathrm{C}\right)$ & 0,922 & $0,920-0,926$ \\
Ácidos graxos livres $(\mathrm{g} / 100 \mathrm{~g}$ de ac. oléico) & $0,3 \%$ & - \\
Índice de peróxidos $(\mathrm{meq} / \mathrm{kg})$ & 1,8 & $3,2-4,5$ \\
Umidade $(\mathrm{g} / 100 \mathrm{~g})$ & 0,3 & \\
Cor Lovibond & 70 amarelo e 1,3 & 1,2 amarelo e \\
& vermelho & $0,1-0,3$ vermelho \\
\hline
\end{tabular}

* Fonte: Weiss (1983).

semelhante ao óleo comercial, sinal de que o processo de prensagem a frio, pode ser uma tecnologia viável em substituição ao processo tradicional de extração de óleo por solvente; a vantagem da prensagem é sua fácil adaptação a pequenos produtores, exigindo baixo investimento inicial.

\section{CONCLUSÕES}

1. O óleo bruto de girassol obtido apresentou características semelhantes àquelas do óleo de girassol comercial.

2. Dentre os parâmetros estudados na prensagem do girassol, os que apresentaram efeito significativo, em ordem decrescente, foram: a umidade com efeito negativo e a interação umidade $\mathrm{x}$ temperatura, também com efeito negativo. $\mathrm{O}$ alto valor do coeficiente de determinação $\left(\mathrm{R}^{2}\right)$, juntamente com um teste $\mathrm{F}$ significativo e um baixo valor do desvio relativo médio, possibilitou a construção da superfície de resposta.

3. O rendimento em óleo bruto variou entre 49 e $65 \%$.

\section{AgRADECIMENTOS}

À FAPESP - Fundação de Amparo à Pesquisa do Estado de São Paulo; ao CNPq - Conselho Nacional de Desenvolvimento Científico e Tecnológico; à UNICAMP - Universidade Estadual de Campinas e ao ITAL - Instituto de Tecnologia de Alimentos.

\section{LITERATURA CITADA}

AOAC - Association of Official Analytical Chemists. Official methods of analysis of AOAC international. 16.ed. Arlington: AOAC - Inc, 1998. sp.

AOCS - American Oil Chemist's Society. Official methods and recommended practices of the AOCS. Champaign: AOCS, 1998. 1200p.

Barros Neto, B. de; Scarmínio, I. S.; Bruns, R. E. Planejamento e otimização de experimentos. Campinas: UNICAMP, 2003. 299p.

Box, G. E. P.; Hunter, W. G.; Hunter, J. S. Statistics for experimenters. An introduction designs, data analysis and model building. New York: John Wiley \& Sons Inc., 1978,. 672p.

Myers, R. H., Montgomery, D. C. Response surface methodology. New York: John Wiley \& Sons Inc., 2002. p.1 - 17.

Singh, J., Bargale, P. C. Mechanical expression of oil from linseed (Linum usitatissimum L.). Journal of Oilseeds Research, v.7, p.106-110, 1990.

Singh, J., Bargale, P. C. Development of a small capacity double stage compression screw press for oil expression. Journal of Food Engineering, v.43, p.75-82, 2000.

Singh, K. K., Wiesenborn, D. P., Tostenson, K., Kangas, N. Influence of moisture content and cooking on screw pressing of crambe seed. Journal of the American Oil Chemists' Society, v.79, p.165-170, 2002.

Singh, M. S., Farsaie, A., Stewart, L. E., Douglass, L. W. Development of mathematical models to predict sunflower oil expression. Transactions of the ASAE, v.27, p.1190-1194, 1984.

Statistica version 6.0. Data analysis software system. Tulsa, StatSoft, Inc., 2001.

Ungaro, M. R. G. Mercados potenciais para o girassol e os seus subprodutos. In: Câmara, G. M. S.; Chiavegato, E. J (ed.). O agronegócio das plantas oleaginosas: Algodão, amendoim, girassol e mamona. Piracicaba: ESALQ, 2001. p.12 -140.

Weiss, E. A. Oilseed processing and products. In: Weiss, E. A. (ed.). Oilseed crops. London: Longman, 1983. cap. 11, p.528-596. 